\title{
Development and Validation of a Discriminating Dissolution Method for Darifenacin Extended-Release Tablets
}

\author{
Leonardo Z. Meneghini', Vinícius Bicca', César A. Junqueira', \\ Andréa I. H. Adams ${ }^{2}$, Graciela Carlos ${ }^{1}$, Pedro E. Fröehlich ${ }^{1}$, and \\ Ana M. Bergold ${ }^{1, *}$ \\ 'Programa de Pós-Graduação em Ciências Farmacêuticas, Faculdade de Farmácia, \\ Universidade Federal do Rio Grande do Sul-UFRGS. Av. Ipiranga, 2752, CEP 906610-000, Porto Alegre, RS, Brasil \\ ${ }^{2}$ Universidade Federal de Santa Maria-UFSM, Centro de Ciências da Saúde, Depto. Farmácia Industrial-Prédio 26, sala 1106, \\ Santa Maria, RS, Brasil
}

\begin{abstract}
A dissolution test for darifenacin 15-mg extended-release tablets was developed and validated according to current FDA and USP guidelines. The sink condition was evaluated, and a $2^{4-1}$ factorial design was employed for method development. In vivo data were obtained from the literature, and the fraction and percentage of dose absorbed (FA) was calculated using the Wagner-Nelson method. The best dissolution conditions were achieved using USP Apparatus 1 (basket) with $900 \mathrm{~mL}$ of dissolution medium containing $2 \%$ SDS at $50 \mathrm{rpm}$. A successful linear regression model of fraction of drug absorbed versus dissolved was achieved $\left(R^{2}=0.9997, p<0.05\right)$. The best fit for drug release kinetics was provided by the Korsmeyer-Peppas model. The validation was performed with an HPLC-UV method, and results for specificity, linearity, precision, and accuracy were in accordance with guidelines. The proposed method achieved a Level A correlation with the fraction of dose absorbed. Additionally, the discriminatory power of the method was challenged. The results show that the proposed test is adequate to evaluate the in vitro profile for extended-release darifenacin tablets.
\end{abstract}

KEYWORDS: Dissolution, darifenacin, extended-release tablet, in vitro-in vivo correlation, factorial design.

\section{INTRODUCTION}

$\mathrm{R}$ ecent years have seen great advances focused increasingly on dissolution testing involving in vitroin vivo correlation (IVIVC) (1). This is due to its usefulness as a potential surrogate for in vivo performance, assisting in decisions concerning the need for bioavailability studies, rendering detection feasible when biopharmaceutical problems occur due to changes in drug product storage conditions and the manufacturing process (2). There are different levels of correlations, and Level A is the highest category of IVIVC. It represents a point-to-point relationship between the in vitro dissolution rate and the in vivo input rate of the drug in the dosage form. The Biopharmaceutics Classification System (BCS) deals with drug solubility and permeability and their influence on the dissolution profile of a drug product, working as a guide to determine the most adequate correlation level that can be established (3-6).

Anticholinergic drugs are commonly used in patients with an overactive bladder for whom conservative management is not applicable (7). Several antimuscarinic drugs are on the market. One such drug is darifenacin, chemically (S)-2-\{1-[2-(2,3-dihydrobenzofuran-5-yl)ethyl]3-pyrrolidinyl\}-2,2-diphenylacetamide hydrobromide, with a molecular weight of 507.5 (Figure 1). It is a potent muscarinic receptor antagonist, available as a hydrobromide

${ }^{*}$ Corresponding author. salt in Enablex, an extended-release tablet that contains $7.5 \mathrm{mg}$ or $15 \mathrm{mg}$ of the drug (8). Pharmacokinetic studies have shown that the drug is rapidly and completely $(\approx 97 \%)$ absorbed following oral administration; it is lipophilic and has a large volume of distribution. The absorption from drug product is limited by the rate of drug release from the tablet matrix, resulting in delayed absorption (9).

The aim of this work was to develop and validate a dissolution test for the Enablex extended-release tablet based on in vivo data. Furthermore, the method was evaluated with expired drug product, and model-dependent approaches were used to explain the dissolution kinetics.

\section{MATERIALS AND METHODS \\ Materials}

Darifenacin hydrobromide (99.70\%) was purchased from Waterstone Technology (USA). Enablex $15 \mathrm{mg}$ was purchased from the national market. The excipients used in the formulation (hypromellose, magnesium stearate, dibasic calcium phosphate, and Opadry white, yellow, and red) were purchased from different suppliers. Analytical grade sodium hydroxide, sodium phosphate, sodium acetate, orthophosphoric acid, acetic acid, and hydrochloric acid were obtained from Merck (Germany). Sodium dodecyl sulfate analytical grade was from Vetec (Brazil). Acetonitrile HPLC grade was from J.T. Baker (USA). The $0.45-\mu \mathrm{m}$ nylon filters were purchased from Millipore (USA), and probe filters $(10 \mu \mathrm{m})$ were from Varian. 


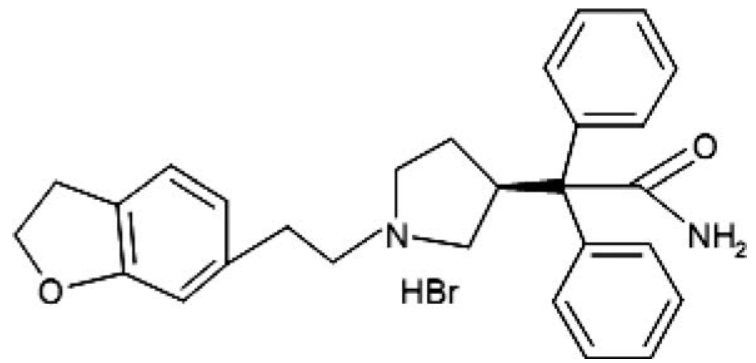

Figure 1. Chemical structure of darifenacin hydrobromide (molecular weight 507.5).

\section{Statistical Analysis and Mathematical Modeling}

The fitted curves from in the vivo study and release kinetics evaluations were performed using Scientist v.2.01 software (Micromath, St. Louis, MO). Design-Expert software (v. 5.0, Stat-Ease, Inc., Minneapolis, MN) was used for factorial fractioned $2^{4-1}$ analysis. Minitab v.15.1.0.0 (Minitab, Inc., State College, PA) was used for statistical analysis in the validation stage.

\section{In Vivo Study}

A one-compartment open model was used to fit a curve using as inputs the average plasma concentration versus time:

$$
C=\frac{F D k_{a}}{V_{d}\left(k_{a}-k_{e}\right)}\left(e^{-k_{e} t}-e^{-k_{a} t}\right)
$$

where $C$ is the plasma concentration at time $t, k_{e}$ is the elimination rate constant, $k_{a}$ is the absorption rate constant, $V_{d}$ is the volume of distribution, $D$ is the dose, and $F$ is the fraction of dose absorbed. In accordance with the guidelines, the in vivo absorption was estimated using the Wagner-Nelson method $(10,11)$.

\section{In Vitro Study}

\section{Dissolution Test Conditions}

The dissolution test was developed and validated using a VANKEL VK 8000 dissolution autosampling station consisting of a VK type bidirectional peristaltic pump, VK 750D digitally controlled heater/circulator, VK 7010 dissolution testing station multi-bath $(n=8)$ with automated sampling manifold. Dissolution was performed using $900 \mathrm{~mL}$ of dissolution medium preheated to $37 \pm 0.5^{\circ} \mathrm{C}$. Sample aliquots were withdrawn at 2, 4, 6, 8, 10, 12, 17, and $24 \mathrm{~h}$ and replaced with an equal volume of fresh medium to maintain a constant total volume.

\section{HPLC Analysis}

The HPLC system consisted of a Shimadzu LC (Kyoto, Japan) composed of an LC-10AD pump, an SPD-M10ADVP photodiode array (PDA) detector, an SLA-10ADVP system controller, a DGU-14A degasser, a CTO-10AS thermostatted column oven, and an SIL-10AD autoinjector. Data were acquired and processed using CLASS-VP software (version 6.1). Chromatographic analysis was done using a Merck $C_{8}$ column $(120 \mathrm{~mm} \times 4.6 \mathrm{~mm}$ i.d., 5 - $\mu \mathrm{m}$ particle size). The mobile phase consisted of a mixture of sodium acetate buffer/acetonitrile (50:50, v/v), pH 5.60. The flow rate was $1.0 \mathrm{~mL} / \mathrm{min}$, the column was equilibrated at $25 \pm 2{ }^{\circ} \mathrm{C}$, and the injection volume was $20 \mu \mathrm{L}$. Detection was carried out by ultraviolet absorption at $210 \mathrm{~nm}$.

\section{Solubility}

Darifenacin hydrobromide solubility was investigated using $\mathrm{BCS}$ recommendations $(4,10,12)$. For this purpose, the sink condition was determined using $0.01 \mathrm{M} \mathrm{HCl}, \mathrm{pH} 6.8$ phosphate buffer, pH 4.5 acetate buffer, and $0.5 \%$ sodium dodecyl sulfate (SDS). An amount of darifenacin hydrobromide equivalent to the highest dose strength was added to vessels containing $250 \mathrm{~mL}$ of aqueous media as previously described $(n=3)$. Media were preheated and maintained at $37.0 \pm 1.0^{\circ} \mathrm{C}$. Rotation speed was set at $100 \mathrm{rpm}$, and samples were collected after 1 and $2 \mathrm{~h}$. Ten-milliliter samples were collected and filtered, neutralized when necessary, diluted with mobile phase, and analyzed by HPLC.

\section{Method Development}

An initial screening was performed using Apparatus 2 (paddle) and different media. Later, a strategy of experimentation using a $2^{4-1}$ factorial fractioned design was applied to obtain better conditions to establish IVIVC with fewer experiments. For four or more factors, it is usually not necessary to run all possible combinations of factor levels (13). Thus, a fractional factorial design was performed varying four factors in two levels with only one set of eight runs (instead of the original 16 runs of full factorial design $2^{4}$ ). Here a run is considered a dissolution profile using the combination between factors and levels from the factorial design. Coefficient of determination $\left(R^{2}\right)$ and model-independent evaluation were used as responses in statistical analysis.

\section{In Vitro-In Vivo Correlation}

The degree of linear relationship between the mean percentage of dose absorbed (FA\%) and the mean percentage of drug dissolved (FD\%) was measured by the Pearson product-moment correlation coefficient (14). For this analysis, a linear mathematical model was considered.

\section{Validation of the Dissolution Procedure}

The most adequate method obtained in the development stage was validated according to current guidelines $(11,15)$.

Specificity

Specificity was evaluated by preparing samples of placebo. The placebo consisted of all the excipients in 
appropriate proportions (hypromellose, magnesium stearate, dibasic calcium phosphate, and Opadry white, yellow, and red) calculated for a medium tablet content weight ( $\approx 215 \mathrm{mg}$ ). The placebo samples were transferred to separate vessels $(n=3)$, filled with $900 \mathrm{~mL}$ of dissolution medium at $37 \pm 0.5^{\circ} \mathrm{C}$ and stirred for $24 \mathrm{~h}$ at $150 \mathrm{rpm}$ using a basket (Apparatus 1). Aliquots were withdrawn and analyzed by HPLC.

\section{Linearity}

A stock solution containing $200 \mu \mathrm{g} / \mathrm{mL}$ of darifenacin was prepared in methanol and diluted with dissolution medium to prepare solutions at seven concentrations ranging from 1.00 to $26.00 \mu \mathrm{g} / \mathrm{mL}$. Ordinary least squares analysis was applied to calibration curve construction $y=m x \pm b$, where $y$ is the response (peak area), $x$ is the concentration, and $m$ and $b$ are the slope and intercept, respectively. The linear model was evaluated by significance of the coefficient of determination $\left(R^{2}\right)$, lack-of-fit, and residual analysis. The solutions were injected in triplicate every day for three consecutive days. Additionally, the limit of quantification (LOQ) was determined based on the signal-to-noise ratio $\left(R_{\mathrm{S} / \mathrm{N}}\right.$ with 10:1 ratio) from a blank injection.

\section{Accuracy and Precision}

Method accuracy was evaluated by the recovery of known amounts of reference substance added to placebo. Aliquots of $0.450,2.70$, and $4.50 \mathrm{~mL}$ of a $4 \mathrm{mg} / \mathrm{mL}$ standard solution dissolved in methanol were added to vessels containing dissolution medium for a final volume of $900 \mathrm{~mL}$ (final concentrations were 2.00, 12.00, and 20 $\mathrm{mg} / \mathrm{mL}$, respectively), preheated at $37^{\circ} \mathrm{C}$ and rotated for $24 \mathrm{~h}$ at $150 \mathrm{rpm}$ using a basket (Apparatus 1). To evaluate the precision, the recovery of the added drug substance was determined on three different days ( $n=3$ for each concentration). The intra- and interday precision was established based on the relative standard deviation (RSD) of the results. Placebo samples were prepared in the same way described in the specificity test.

\section{Stability Study}

A stability study was performed using drug substance and drug product. The solutions were kept at $37 \pm 2{ }^{\circ} \mathrm{C}$ for $24 \mathrm{~h}$ under light shaking and were then left at room temperature, tightly closed, for $24 \mathrm{~h}$ (overall assay time $48 \mathrm{~h}$ ).

Aliquots of the samples were tested at time 0 and after 24 and $48 \mathrm{~h}$. The responses for aged solutions were evaluated using a freshly prepared standard. The assay was performed in triplicate.

\section{Evaluation of Release Kinetics}

The release kinetics was evaluated using the experimental dissolution profile according to five different models (Table 1): zero-order, first-order, Higuchi, Hixson-Crowell, and Korsmeyer-Peppas (16). The most appropriate model was selected based on the coefficient of determination
$\left(R^{2}\right)$ and model selection criteria (MSC). The latter is a modified Akaike Information Criterion; the most appropriate model will be that with the largest MSC value.

\section{Evaluation of Dissolution Profiles}

The discriminatory power of the proposed dissolution test was evaluated. For this, a model-independent approach was used for comparison with expired darifenacin hydrobromide tablets. This analysis includes the difference factor $\left(f_{1}\right)$ and the similarity factor $\left(f_{2}\right)$.

The $f_{1}$ measures the percent significant difference between two curves over all time points:

$$
f_{1}=\left\{\left[\sum_{t=1}^{n}\left|R_{t}-T_{t}\right|\right] /\left[\sum_{t=1}^{n} R_{t}\right]\right\} \times 100
$$

where $n$ is the number of time points and $R_{t}$ and $T_{t}$ are the percent dissolved of the reference and test products, respectively, at each time point. The percent error is zero when the test and drug reference profiles are identical and increases proportionally with the dissimilarity between the two dissolution profiles (17). The $f_{2}$ factor is a logarithmic transformation of the sum-squared error of differences between the test and the reference products over all time points:

$$
f_{2}=50 \cdot \log \left\{\left[1+\frac{1}{n} \sum_{t=1}^{n}\left(R_{t}-T_{t}\right)^{2}\right]^{-0.5} \times 100\right\}
$$

This factor is 100 when the test and reference profiles are identical and moves toward 0 as the similarity decreases (17). According to the FDA, two dissolution profiles are declared similar if $f_{1}$ is between 0 and 15 and if $f_{2}$ is between 50 and $100(10)$.

Table 1. Models Used in the Assessment for Release Kinetics

\begin{tabular}{ll}
\hline \multicolumn{1}{c}{ Model } & \multicolumn{1}{c}{ Equation } \\
\hline Zero-order kinetics & $Q_{t}=Q_{0}+K_{0} t$ \\
\hline First-order kinetics & $\log Q_{t}=\log Q_{0}+\left(K_{1} t\right) / 2.303$ \\
\hline Higuchi & $f_{t}=K_{H} t^{1 / 2}$ \\
\hline Hixson-Crowell & $W_{0}^{1 / 3}-W_{t}^{1 / 3}=K_{s} t$ \\
\hline Korsmeyer-Peppas & $M_{t} / M_{\infty}=a t^{n}$ \\
\hline
\end{tabular}

$Q_{t}$ : amount of drug dissolved in time $t$

$Q_{0}$ : initial amount of drug in the solution

$K_{0}$ and $K_{1}$ : zero-order and first-order release constants

$f_{t}$ : amount of drug released in time $t$ by surface unity

$K_{H}$ : Higuchi dissolution constant

$W_{0}$ : initial amount of drug in the pharmaceutical dosage form

$W_{t}$ : remaining amount of drug in the pharmaceutical dosage form at time $t$ $K_{s}$ : constant incorporating the surface-volume relation

$M_{t} / M_{\infty}$ : fractional release of drug as a function of time $t$ a: constant incorporating structural and geometrical characteristics of drug dosage form

n: release exponent 


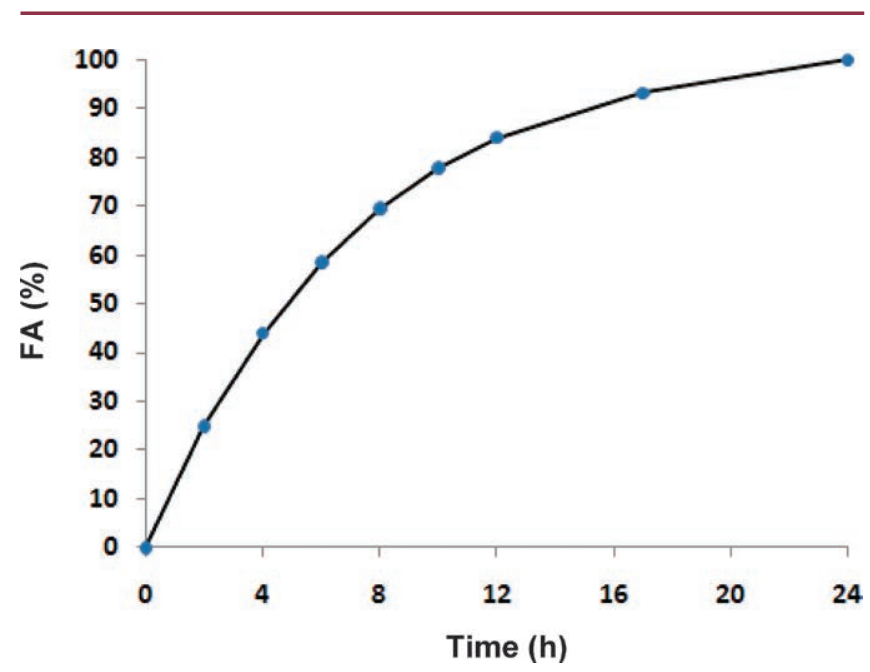

Figure 2. Percentage of dose absorbed vs. time of darifenacin extendedrelease tablet $15 \mathrm{mg}$ using the Wagner-Nelson method.

\section{RESULTS AND DISCUSSION \\ Solubility Determination}

The results for the darifenacin solubility evaluation demonstrate that drug product is soluble in $0.01 \mathrm{M} \mathrm{HCl}$, pH 6.8 phosphate buffer, $\mathrm{pH} 4.5$ acetate buffer, and $0.5 \%$ sodium dodecyl sulfate. According to the BCS and the literature about the permeability study $(9,17)$, the drug can be classified as Class 1 (high solubility-high permeability). In addition, the sink condition is necessary to provide appropriate media without the interference of drug solvent saturation phenomena. Dissolution method development was accomplished considering these features.

\section{Method Development}

The plasma concentration-versus-time profile obtained from the literature (9) was transformed into percentage of drug absorbed $(F A)$ versus time using the Wagner-Nelson method (Figure 2). The estimation of the in vivo absorption or dissolution time using an appropriate deconvolution technique (e.g., Wagner-Nelson, Loo-Riegelman,

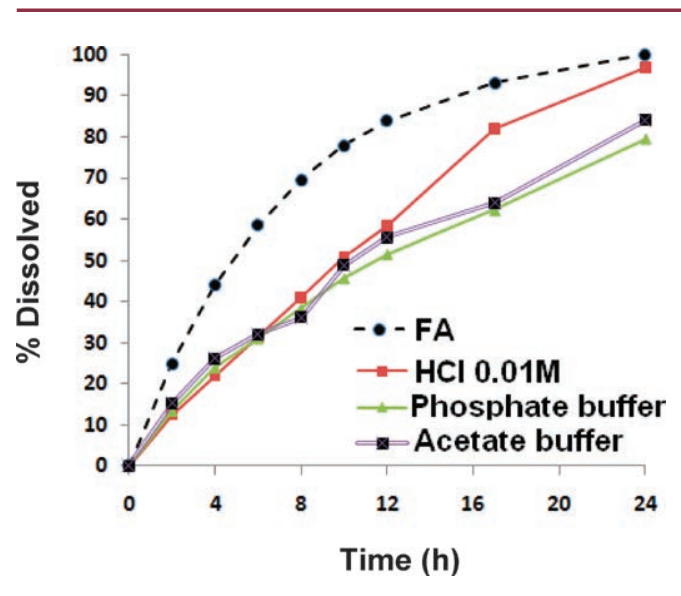

Figure 3. Dissolution profiles performed with $900 \mathrm{~mL}$ medium volume, Apparatus 2 (paddle), at $50 \mathrm{rpm}$.

numerical deconvolution) is recommended in guidelines for IVIVC studies $(10,11)$.

The initial screening was attempted with $0.01 \mathrm{M}$ hydrochloric acid, pH 4.5 acetate buffer, and pH 6.8 phosphate buffer $(900 \mathrm{~mL}$ ) using Apparatus 2 with a 50 -rpm rotation speed (Figure 3 ). The regression analysis of FD versus FA did not show an adequate determination coefficient (about 0.90 for all media; information not shown). Subsequently a thorough evaluation was carried out with a fractioned factorial design (Table 2, Figure 4) using apparatus type, medium type, medium concentration, and rotation speed as factors. Coefficient of determination $\left(R^{2}\right)$ and model-independent evaluations (by $f_{1}$ and $f_{2}$ ) were used as responses to choose the most adequate dissolution conditions for IVIVC evaluations. Here, a model-independent approach was applied to estimate the similarity between dissolution FA profile and FD profiles (runs).

The results demonstrate that the conditions used in run 7 (Figures $4 \mathrm{~b}$ and 5) were the most satisfactory. Furthermore,

Table 2. Experimental Matrix Used in Fractioned Factorial Design $2^{4-1}$

\begin{tabular}{|c|c|c|c|c|c|c|c|}
\hline Run & Apparatus & Medium & Concentration (\%) & Rotation (rpm) & $R^{2}$ & $f_{1}$ & $f_{2}$ \\
\hline 1 & Basket & $0.1 \mathrm{M} \mathrm{HCl}$ & 50 & 50 & 0.9417 & 45.74 & 27.64 \\
\hline 2 & Paddle & $0.1 \mathrm{M} \mathrm{HCl}$ & 50 & 75 & 0.8913 & 36.51 & 32.44 \\
\hline 3 & Basket & $2 \%$ SDS & 50 & 75 & 0.9802 & 17.71 & 48.28 \\
\hline 4 & Paddle & $2 \%$ SDS & 50 & 50 & 0.9764 & 17.09 & 47.80 \\
\hline 5 & Basket & $0.1 \mathrm{M} \mathrm{HCl}$ & 100 & 75 & 0.9207 & 43.67 & 28.38 \\
\hline 6 & Paddle & $0.1 \mathrm{M} \mathrm{HCl}$ & 100 & 50 & 0.9170 & 36.45 & 32.71 \\
\hline 7 & Basket & $2 \%$ SDS & 100 & 50 & 0.9997 & 0.48 & 96.71 \\
\hline 8 & Paddle & $2 \%$ SDS & 100 & 75 & 0.9079 & 14.57 & 51.46 \\
\hline
\end{tabular}



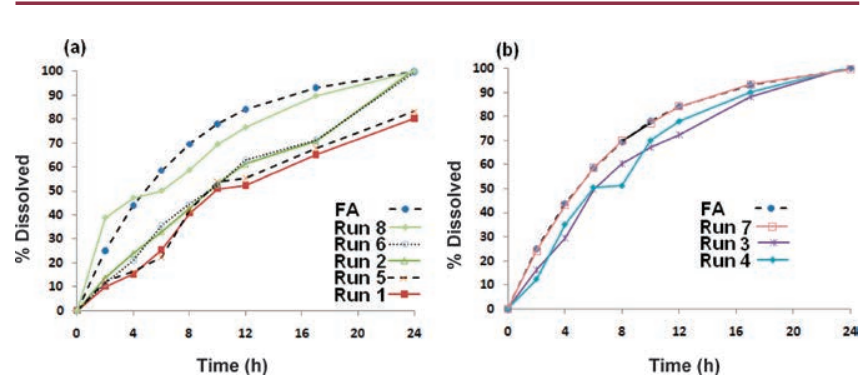

Figure 4. Dissolution profiles (runs) obtained in the conditions from $2^{4-1}$ experimental design matrix and FA profile (in vivo study; ordinate axis is absorbed percentage).
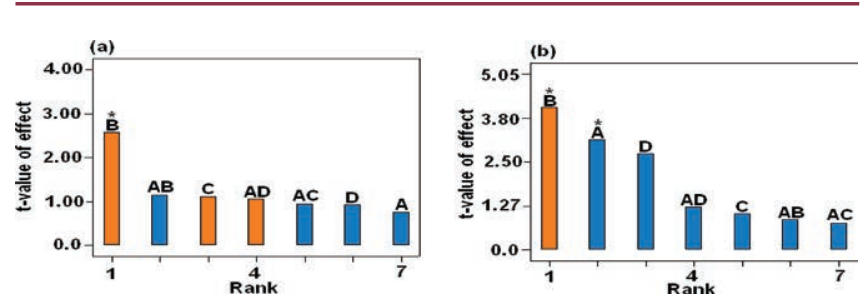

Figure 5. Pareto charts showing statistical analysis $(\alpha=0.05)$ for the responses (a) similarity factor, $f_{2}$, and (b) determination coefficient, $R^{2}$; factors are presented as $A, B, C, D$ for apparatus type, medium type, medium concentration, and speed rotation, respectively. The interaction between the factors is presented as $A B, A C$, and $A D$.

the dissolution profiles with Apparatus 2 presented higher RSD values than those using Apparatus 1 (about 20\% and $2 \%$ for paddle and basket, respectively; information not shown) in all design runs. Medium composition is the main factor (B) influencing the $R^{2}$ and $f_{2}\left(f_{1}\right.$ presented the same result; information not shown) dissolution profile by Pareto chart analysis (Figure 5), with better results when SDS is used. In addition, apparatus type influences regression analysis with higher values for $R^{2}$ using the basket.

Although this kind of experimental strategy presents many advantages for the dissolution study $(18,19)$, sometimes a typical factorial design statistic is not able to detect low magnitude levels of model variance $(20,21)$. Thus, the use of $2 \%$ SDS is more suitable than $1 \%$ SDS although statistical analysis has not been considered a significant factor.

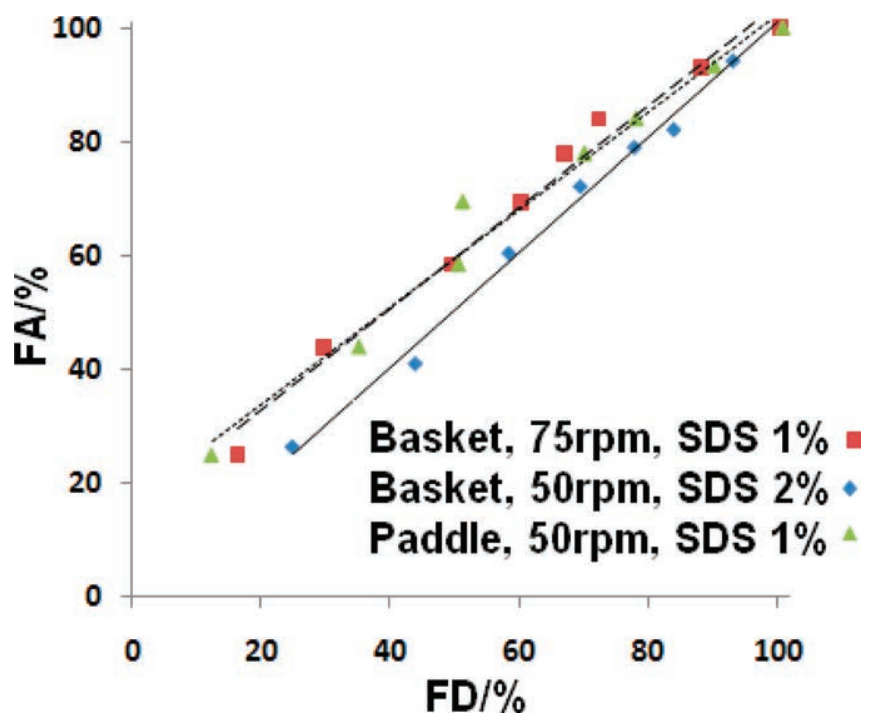

Figure 6. Plot of mean percentage of dose absorbed (FA) versus mean percentage of dose dissolved (FD) for Enablex. The line of best fit is shown for each dissolution medium.

The critical micelle concentration remained above its critical limit at both levels of SDS concentration; the occurrence of some type of phenomenon with a hypromellose structure in the tablet is possible. When the SDS concentration was increased to $2 \%$, the increasing number of micelles possibly modified the polymer structure in the solvated state (involving the transport process, diffusion, and chain disentanglement), decreasing resistance to mass transfer of the drug from a matrix structure to dissolution medium (22-24). In this way, the method was adjusted to increase the tablet release velocity to obtain a profile closer to that of the FA (Figure 4b). Considering the aim of this work, run 7 was designated to be used at later stages because at a higher $R^{2}$, the model will probably be more predictive and biorelevant.

IVIVC

Table 3 and Figure 6 show results for the comparison between the better profiles from factorial design. These results confirm that dissolution conditions for run 7

Table 3. Statistical Analysis for IVIVC Regression

\begin{tabular}{|c|c|c|c|c|c|c|}
\hline \multirow[t]{2}{*}{ Conditions } & \multirow[t]{2}{*}{ Run } & \multicolumn{2}{|c|}{ Slope $(m)^{a}$} & \multicolumn{2}{|c|}{ Intercept $(b)^{a}$} & \multirow[t]{2}{*}{$R^{2}$} \\
\hline & & value & $p$ value ${ }^{b}$ & value & $p$ value $^{b}$ & \\
\hline Basket, 75 rpm, 1\% SDS & 3 & 0.89 & $<0.001$ & 14.95 & 0.004 & 0.9822 \\
\hline Basket, 50 rpm, 2\% SDS & $7^{c}$ & 0.99 & $<0.001$ & 0.99 & 0.91 & 0.9997 \\
\hline Paddle, 50 rpm, $1 \%$ SDS & 4 & 0.86 & $<0.001$ & 16.79 & 0.003 & 0.9764 \\
\hline
\end{tabular}


(basket, 50 rpm, 2\% SDS) are the most adequate for IVIVC establishment.

\section{Validation of Dissolution Method}

The results of the dissolution method validation are shown in Table 4 and Figure 7. Darifenacin was stable under dissolution test conditions. The results demonstrate that sample and standard solutions remained at $100.0 \pm 2.0 \%$ over a period of $48 \mathrm{~h}$.

Specificity was assessed by photodiode array using the purity computation method (CLASS-VP V6.1 algorithm). The total peak purity mode was selected, and peak overlapping was not detected in the retention time of darifenacin. As shown in Figure 7, no medium or placeborelated peaks were detected at the same retention time as darifenacin. These results demonstrate the specificity of the method.

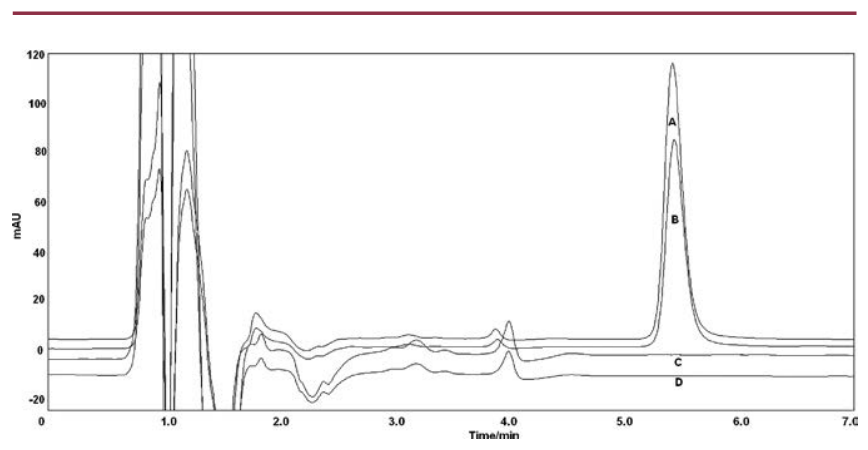

Figure 7. Representative chromatograms showing (A) samples of drug substance, (B) drug product, (C) placebo, and (D) dissolution medium.

Table 4. Requirements Assessed for Method Validation

\begin{tabular}{|c|c|c|c|c|c|c|c|}
\hline \multicolumn{4}{|c|}{ Linearity } & \multicolumn{4}{|c|}{ Accuracy } \\
\hline Parameter & \multirow{2}{*}{$\begin{array}{l}\text { Value } \\
0.999\end{array}$} & Confidence Limit & $p$ value & \multicolumn{2}{|c|}{ Concentration $(\mu \mathrm{g} / \mathrm{mL})$} & \multicolumn{2}{|c|}{ Recovery (\%) } \\
\hline$R^{2}$ & & - & - & \multicolumn{2}{|c|}{2.00} & \multicolumn{2}{|c|}{99.49} \\
\hline$m^{a}$ & 53202 & \pm 828 & 0.55 & \multicolumn{2}{|c|}{12.00} & \multicolumn{2}{|c|}{100.48} \\
\hline$b^{a}$ & -3902 & \pm 13342 & $<0.01$ & \multicolumn{2}{|c|}{20.00} & \multicolumn{2}{|c|}{101.35} \\
\hline \multirow[t]{2}{*}{$\mathrm{LQ}^{b}$} & 0.24 & - & - & & & & \\
\hline & & & & \multicolumn{4}{|c|}{ Precision } \\
\hline \multicolumn{4}{|c|}{ Model assessment } & \multicolumn{2}{|l|}{ Intraday } & \multirow{2}{*}{$\begin{array}{c}\text { Mean }^{c} \\
(\mu \mathrm{g} / \mathrm{mL})\end{array}$} & \multirow{2}{*}{$\begin{array}{r}\text { RSD } \\
(\%)\end{array}$} \\
\hline & & Val & & Level & Day & & \\
\hline \multirow[t]{3}{*}{ Parameter } & Statistical test & Critical & Calculated & 2.00 & 1 & 99.26 & 1.15 \\
\hline & & & & & 2 & 98.71 & 1.22 \\
\hline & & & & & 3 & 100.49 & 1.51 \\
\hline \multirow[t]{3}{*}{ Regression } & ANOVA $F$ value & 3.87 & 18081 & 12.00 & 1 & 99.85 & 0.64 \\
\hline & & & & & 2 & 100.50 & 0.72 \\
\hline & & & & & 3 & 101.10 & 0.47 \\
\hline \multirow[t]{3}{*}{ Lack-of-fit } & ANOVA $p$ value & 0.05 & 0.73 & 20.00 & 1 & 100.85 & 0.85 \\
\hline & & & & & 2 & 101.19 & 0.58 \\
\hline & & & & & 3 & 102.01 & 0.68 \\
\hline \multirow[t]{3}{*}{ Autocorrelation } & $D^{d}-W$ & $d_{L}=1.22$ & 2.28 & & & & \\
\hline & $D$ value & $d_{U}=1.42$ & & Intraday & & & \\
\hline & & & & $\begin{array}{c}\text { Level } \\
(\mu \mathrm{g} / \mathrm{mL})\end{array}$ & & $\begin{array}{c}\text { Meanc } \\
(\mu \mathrm{g} / \mathrm{mL})\end{array}$ & $\begin{array}{l}\text { RSD } \\
(\%)\end{array}$ \\
\hline Homoscedasticity & Levene $p$ value & 0.05 & 0.55 & 2.00 & & 99.49 & 0.92 \\
\hline \multirow[t]{2}{*}{ Normality test } & $A^{e}-D p$ value & 0.05 & 0.43 & 12.00 & & 100.48 & 0.62 \\
\hline & & & & 20.00 & & 101.35 & 0.59 \\
\hline \multicolumn{8}{|c|}{$\begin{array}{l}a y=m x+b \\
{ }^{b} \text { Limit of Quantification } \\
{ }^{c} n=3 \\
{ }^{d} \text { Durbin-Watson statistic } \\
{ }^{e} \text { Anderson-Darling statistic }\end{array}$} \\
\hline
\end{tabular}


Other relevant elements of the validation study (Table 4) show that the method presents low variability and high accuracy and precision in accordance with the literature (11).

\section{Evaluation of Release Kinetics}

Mathematical models describing polymer behavior like that of hypromellose in solution have been studied extensively using various mechanisms and models to characterize dissolution behavior (25). Table 5 shows the evaluation of release kinetics comparing $R^{2}$ and MSC among various mathematical models. The Korsmeyer-Peppas model proved the most adequate. This model presents an exponential equation where the $n$ exponent depends on the polymer swelling characteristics and relaxation rate at the swelling front, as demonstrated in Table 6. The dissolution data fit according to the model results in $n=0.7344$, indicating superposition of two phenomena: diffusion

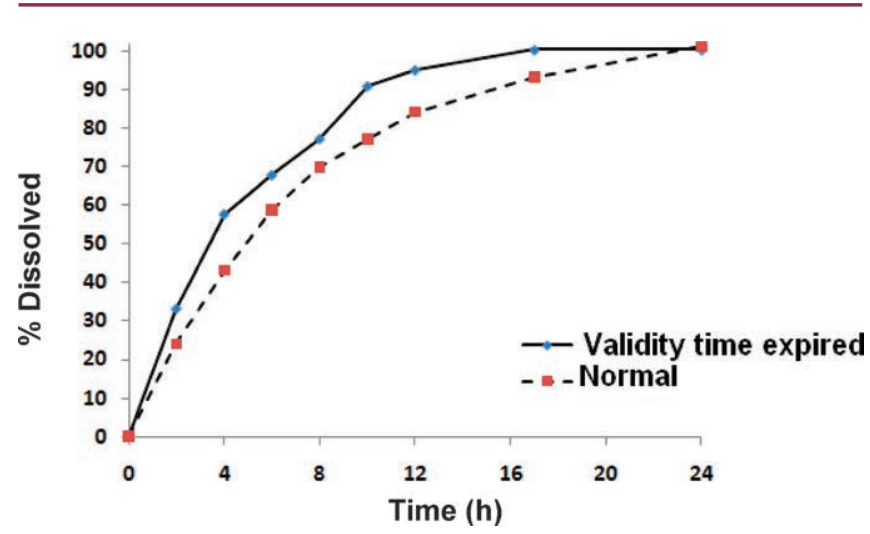

Figure 8. Changes in the dissolution rate of darifenacin tablet after date expired.

Table 5. Analysis of the Release Kinetics of Different Models

\begin{tabular}{ccc}
\hline Model & $\boldsymbol{R}^{\mathbf{2}}$ & MSC \\
\hline Zero-order & 0.9792 & 1.32 \\
\hline First-order & 0.8783 & -2.70 \\
\hline Higuchi & 0.9134 & 0.56 \\
\hline Hixson-Crowell & 0.9481 & 0.45 \\
\hline Korsmeyer-Peppas & 0.9953 & 4.37 \\
\hline
\end{tabular}

(Fickian behavior) and swelling-controlled drug release (26). This result corroborates other studies (27) dealing with extended-release systems containing water-soluble drugs.

\section{Discriminating Power of the Test}

When the intention is to perform a dissolution test with IVIV, dosage form modifications should be detected in the dissolution profile because functional modification in a formulation may alter drug bioavailability $(28,29)$. Figure 8 shows comparative dissolution profiles between a time-expired tablet and non-expired tablet. The modelindependent analysis supplied the values of 17.67 and 48.67 for $f_{1}$ and $f_{2}$, respectively. These measurements indicate that the dissolution profiles were not similar, and the dissolution test proposed could point to a difference in the expiration date of the formulation.

\section{CONCLUSIONS}

A Level A in vitro correlation was established for darifenacin hydrobromide. The in vitro dissolution profile was obtained using Apparatus 1 (basket) with $900 \mathrm{~mL}$ of dissolution medium containing $2 \%$ SDS at $50 \mathrm{rpm}$ and $37 \pm 0.5^{\circ} \mathrm{C}$. Adequate specificity, linearity, precision, and accuracy were obtained in the validation stage. In vitro profiles for darifenacin tablets were successfully evaluated by the dissolution method proposed.

\section{REFERENCES}

1. Azarmi, S.; Roa, W.; Löbenberg, R. Current perspectives in dissolution testing of conventional and novel dosage forms. Int. J. Pharm. 2007, 328 (1), 12-21.

2. Wang, Q.; Fotaki, N.; Mao, Y. Biorelevant Dissolution: Methodology and Application in Drug Development. Dissolution Technol. 2009, 16 (3), 6-12.

3. Waiver of In Vivo Bioavailability and Bioequivalence Studies for Immediate-Release Solid Oral Dosage Forms Based on a Biopharmaceutics Classification System; Guidance for Industry; U.S. Department of Health and Human Services, Food and Drug Administration, Center for Drug Evaluation and Research (CDER), U.S. Government Printing Office: Washington, DC, 2000.

4. Basanta, B.; Reddy, K.; Karunakar, A. Biopharmaceutics Classification System: A Regulatory Approach. Dissolution Technol. 2011, 18 (1), 31-37.

Table 6. Variation of $\boldsymbol{n}$ Values with a Drug Release Mechanism in the Korsmeyer-Peppas Model

\begin{tabular}{ccc}
\hline $\boldsymbol{n}$ & Mechanism & $\boldsymbol{d} \boldsymbol{M}_{\boldsymbol{t}} / \boldsymbol{d t}$ dependence \\
\hline 0.5 & Fickian diffusion & $t^{0.5}$ \\
\hline $0.5<n<1.0$ & Anomalous diffusion & $t^{n-1}$ \\
\hline 1.0 & Case II transport & Zero order \\
\hline$n>1.0$ & Super Case Il transport & $t^{n-1}$ \\
\hline
\end{tabular}


5. Sirisuth, N.; Eddington, N. D. In-Vitro-In-Vivo Correlation Definitions and Regulatory Guidance. Int. J. Generic Drugs 2002, 19, 1-11.

6. Demrtürk, E.; Öner, L. In Vitro-In Vivo Correlations. FABAD J. Pharm. Sci. 2003, 28, 215-224.

7. Novara, G.; Galfano, A.; Secco, S.; D'Elia, C.; Cavalleri, S.; Ficarra, V.; Artibani, W. A Systematic Review and Meta-Analysis of Randomized Controlled Trials with Antimuscarinic Drugs for Overactive Bladder. Eur. Urol. 2008, 54 (4), 740-764.

8. Chapple, C.; Steers, W.; Norton, P.; Millard, R.; Kralidis, G.; Glavind, K.; Abrams, P. A pooled analysis of three phase III studies to investigate the efficacy, tolerability and safety of darifenacin, a muscarinic $M_{3}$ selective receptor antagonist, in the treatment of overactive bladder. BJU Int. 2005, 95 (7), 993-1000.

9. Skerjanec, A. The Clinical Pharmacokinetics of Darifenacin. Clin. Pharmacokinet. 2006, 45 (4), 325-350.

10. Extended Release Oral Dosage Forms: Development, Evaluation, and Application of In Vitro/In Vivo Correlations; Guidance for Industry; U.S. Department of Health and Human Services, Food and Drug Administration, Center for Drug Evaluation and Research (CDER), U.S. Government Printing Office: Washington, DC, 1997.

11. The United States Pharmacopeia and National Formulary USP 32-NF 27; The United States Pharmacopeial Convention, Inc.: Rockville, MD, 2009.

12. Löbenberg, R.; Amidon, G. L. Modern bioavailability, bioequivalence and biopharmaceutics classification system: New scientific approaches to international regulatory standards. Eur. J. Pharm. Biopharm. 2000, 50 (1), 3-12.

13. Brereton, R. G. Chemometrics: Data Analysis for the Laboratory and Chemical Plant; Wiley: New York, 2003.

14. Sheskin, D. J. Handbook of Parametric and Nonparametric Statistical Procedures; Chapman \& Hall/ CRC: Boca Raton, FL, 2004.

15. International Conference on Harmonisation of Technical Requirements for Registration of Pharmaceuticals for Human Use. Validation of Analytical Procedures: Text and Methodology, Q2(R1); ICH Harmonised Tripartite Guideline: Geneva, Switzerland, 2005.

16. Costa, P.; Lobo, J. M. S. Modeling and comparison of dissolution profiles. Eur. J. Pharm. Sci. 2001, 13, 123-133.
17. Emami, J. In vitro-In vivo Correlation: From Theory to Applications. J. Pharm. Pharm. Sci. 2006, 9 (2), 169-189.

18. Abrahamsson, B.; Alpsten, M.; Bake, B.; Jonsson, U. E.; Eriksson-Lepkowska, M.; Larsson, A. Drug absorption from a nifedipine hydrophilic matrix extended-release (ER) tablet-comparison with an osmotic pump tablet and effect of food. J. Controlled Release 1998, 52 (3), 301-310.

19. Likar, M. D.; Mansour, H. L.; Harwood, J. W. Development and validation of a dissolution test for a once-a-day combination tablet of immediate-release cetirizine dihydrochloride and extended-release pseudoephedrine hydrochloride. J. Pharm. Biomed. Anal. 2005, 39 (3-4), 543-551.

20. Montgomery, D. C. Design and Analysis of Experiments, 5th ed.; Wiley: Hoboken, NJ, 2001.

21. Mason, R. L.; Gunst, R. F.; Hess, J. L. Statistical Design and Analysis of Experiments; Wiley: Hoboken, NJ, 2003.

22. Holmberg, K.; Jönsson, B.; Kronberg, B.; Lindman, B. Surfactants and Polymers in Aqueous Solution; Wiley: Chichester, U.K., 2002.

23. Narasimhan, B. Mathematical models describing polymer dissolution:consequences for drug delivery. Adv. Drug Deliv. Rev. 2001, 48 (2-3), 195-210.

24. Li, Y.; Chen, X.; Zhang, X.; Zhu, F.; Zhu, Y. Complexation behaviour of cellulose derivative/surfactant mixtures investigated by nonlinear enhanced Rayleigh scattering. Colloid Polym. Sci. 2011, 289 (7), 767-774.

25. Fu, X. C.; Wang, G. P.; Liang, W. Q.; Chow, M. S. S. Prediction of drug release from HPMC matrices: effect of physicochemical properties of drug and polymer concentration. J. Controlled Release 2004, 95 (2), 209216.

26. Siepmann, J.; Peppas, N. A. Modeling of drug release from delivery systems based on hydroxypropyl methylcellulose (HPMC). Adv. Drug Deliv. Rev. 2001, 48 (2-3), 139-157.

27. Conti, S.; Maggi, L.; Segale, L.; Ochoa Machiste, E.; Conte, U.; Grenier, P.; Vergnault, G. Matrices containing $\mathrm{NaCMC}$ and HPMC: 1. Dissolution performance characterization. Int. J. Pharm. 2007, 333 (1-2), 136-142.

28. Yoshioka, S.; Stella, V. J. Stability of Drugs and Dosage Forms; Kluwer Academic Publishers: New York, 2002.

29. Rhodes, C. T.; Carstensen, J. T. Drug Stability: Principles and Practices; Marcel Dekker: New York, 2002. 